\title{
Long-Term Efficacy of Repositioning Maneuvers in Benign Paroxysmal Positional Vertigo
}

\author{
Erika Barioni Mantello ${ }^{1,2}$, Ana Paula do Rego André ${ }^{1,2}$, Nathali Singaretti Moreno ${ }^{1}$, \\ Miguel Ângelo Hyppolito ${ }^{1}$, Julio Cesar Moriguti ${ }^{3}$
}

${ }^{1}$ Department of Ophthalmology, Otorhinolaryngology and Head and Neck Surgery, Faculty of Medicine of Ribeirão Preto, University of São Paulo, Ribeirão Preto, Brazil (HCFMRP-USP); ${ }^{2}$ University of Franca, São Paulo, Brazil; ${ }^{3}$ Department of Internal Medicine, Faculty of Medicine of Ribeirão Preto, University of São Paulo, Ribeirão Preto, Brazil (HCFMRP-USP).

Email: mahyppo@fmrp.usp.br

Received September $3^{\text {rd }} 2010$; revised January $17^{\text {th }} 2011$; accepted January $18^{\text {th }} 2011$.

\begin{abstract}
To determine the characteristics of 12 patients with a diagnosis of benign paroxysmal positional vertigo (BPPV) treated by the repositioning maneuver during a 24-month follow-up period after the initial discharge a longitudinal clinical study was performed in which a questionnaire for the assessment of discomfort caused by dizziness was applied at the first visit, at the last visit after treatment and 2 years after the initial discharge. Repositioning treatment was again offered to patients who suffered relapses. In 10 cases (83.34\%) there was no recurrence of symptoms and 2 patients (16.66\%) presented symptoms exactly 2 years after the original discharge showing that treatment of BPPV by repositioning manuevers is effective in the long term, but new studies in larger populations are needed to determine the pattern of the rates of recurrence of $B P P V$.
\end{abstract}

Keywords: Positional Vertigo, Dizziness, Recurrence Omponen

\section{Introduction}

Benign paroxysmal positional vertigo (BPPV) is a frequent peripheral dysfunction of the vestibular system characterized by brief episodes of vertigo when the head is moved in certain positions. In addition to rotary dizziness, this causes positional nausea, vomiting, sudoresis, disequilibrium, tinnitus and sometimes hearing loss [1]. BPPV occurs when degenerated calcium carbonate particles of the utricular otoconia are dislodged by the action of gravity and by movement of the head, remaining suspended in the semicircular canals (ductolithiasis) or adhering to their cupula (cupulolithiasis) [2]. The causes of this conditions are varied, one of them being traumatic injury to the skull; however, most cases are of idiopathic origin.

Recent knowledge about the physiopathology of BPPV has led to different treatment approaches, with repositioning maneuvers gaining more space due to the high cure rate and the short treatment time. These maneuvers can reposition the otoliths back into the utricle, whereas medications have no effect on repositioning, simply treating the neurovegetative symptoms inherent to the peripheral vestibular signs and symptoms. In some cases the patients are discharged within one week, after a single repositioning maneuver [3].

Repositioning maneuvers are an alternative type of treatment for individuals with positional dizziness (the type most frequently detected) or chronic or central dizziness or dizziness occurring in bouts. Until recently, repositioning maneuvers were strictly associated with vestibular rehabilitation (VR), a program of physical exercises associated with a set of measures and changes in habits aiming at the acceleration of vestibular compensation. VR is an important and effective strategy for the treatment of individuals with disorders of bodily equilibrium, providing a marked improvement of quality of life [3]. Studies of VR in general, and specifically of BPPV, conducted in recent years in Brazil as well as in other countries are limited to the description of patient follow-up after discharge and do not provide information about recurrence [4-8].

Bittar et al. have described risk factors for patients in whom repositioning treatment fails or who experience recurrences [2]. Some of these factors are: inappropriate indication, vasomotor instability, physical limitations, psychiatric problems, and traumatic skull-brain injuries [2]. Epley reported a 30\% to $45 \%$ recurrence rate among 
patients [9]. There is great interest in this topic, as demonstrated by the large number of published papers and investigations conducted both in Brazil and at university centers all over the world regarding the maneuvers of BPPV treatment. However, few studies have reported on patient follow-up over a long period of time after discharge in order to determine possible recurrences of the disease, which would permit a longitudinal view of the real therapeutic efficacy of this approach to BPPV rehabilitation.

Cohen and Jerabeck detected an almost null rate of recurrence of symptoms in a population of 87 patients after a 6 month follow-up regarding the frequency and intensity of vertigo [10]. Hain et al. detected a $17 \%$ rate of recurrence during the first four to six months after treatment, increasing to $47 \%$ among patients treated with VR after 5 years of follow-up [11]. Sidhar and Panda detected recurrences in $5 \%$ of their patients six months after treatment, with an increase to $10 \%$ one year after treatment [5].

Although there is consensus in the literature that the Epley maneuver is an excellent method for the treatment of BPPV for the vertical canal, new studies are needed to investigate vestibular suppressors and surgical treatment, as well as an extended patient follow-up since the maneuver does not guarantee complete long-term resolution of symptoms [11]. Although many studies have confirmed the therapeutic efficacy of this method, especially in BPPV, few authors have commented about the possibility of recurrence [2-8,10-17]. Thus, it is important to follow up patients submitted to this modality of treatment in order to determine whether the repositioning maneuvers used for BPPV were actually effective on a longterm basis. This is also important in order to define a new conduct in cases of relapse and to determine whether VR contributed to a second etiologic diagnosis leading to a new treatment of vertigo.

The general objective of the present study was to follow up 12 patients diagnosed with BPPV and treated with the repositioning maneuver during the first 24 months after discharge, and the specific objectives were to determine the occurrence of relapses and to characterize relapsing patients with labyrinth symptoms.

\section{Material and Methods}

The study was conducted on 12 patients, 2 males and 10 females, ranging in age from 22 to 75 years, with a diagnosis of BPPV confirmed by an otorhinolaryngologist and treated for more than 24 months by a speech therapist with repositioning maneuvers. The repositioning maneuvers used for treatment were the Epley maneuver for otolith repositioning and the Brandt-Daroff maneuver for therapeutic complementation in partially asympto- matic cases. The Dix-Hallpike test was used for treatment monitoring and for the detection of recurrences [8,9, 13]. After the first repositioning maneuver, the protocol described in Enclosure I was followed. Vestibulo-visual and proprioceptive exercises were performed. For the phonoaudiologic treatment of VR, exercises were indicated in order to accelerate vestibular compensation. These exercises were performed slowly and gradually at the patient's home, with the patient initially sitting down, in order to stimulate the vestibulo-ocular reflexes (Figure 1), followed by stimulation of the vestibulospinal reflex (Figure 2) [15]. The exercises were performed 3 times a day, with 20 repetitions during each period.

The study was approved by the Research Ethics Committee of the University of Franca (UNIFRAN - protocol no. 162/03) and all patients gave written informed consent sent to participate. Data were obtained from the medical records of the 12 patients with a diagnosis of BPPV treated with repositioning maneuvers from May to July 2002. The patients were called on the telephone and invited to participate in a late follow-up study two years after being submitted to treatment for BPPV. After the patients accepted to participate, they were briefly interviewed about their current symptoms and responded to a questionnaire for the assessment of discomfort concerning the severity of the symptoms on initial visit to mild, moderate, moderately severe, severe, serious or incapacitating and about therapeutic success on final visit to asymptomatic, partial success, relative success, unchanged and Failure (Enclosure II), the same applied immediately after their initial discharge [18].

\section{Results}

Of the 12 patients who participated in the study, 83.34\% ( $n=10)$ had no recurrence of the symptoms related to BPPV over the last two years, whereas $16.66 \%(n=2)$ experienced recurrence of symptoms by 18 months after discharge from the first treatment of BPPV. All the 10 patients with no recurrence of symptoms reported that they had continued to follow dietary guidelines and changes in life habits and to engage in sports activities on a daily basis throughout the period after discharge. Of the two patients who suffered recurrence of symptoms, only one (50\%) continued to follow the suggested guidelines.

During the evaluation conducted before treatment, when the etiologic diagnosis of BPPV was confirmed, $33.33 \%(n=4)$ of the subjects stated that vertigo caused them severe discomfort, $33.33 \%(n=4)$ reported moderately severe discomfort, $25 \%(n=3)$ reported moderate discomfort, and $8.33 \%(n=1)$ reported serious discomfort.

During the evaluation performed one day after the end of treatment, 83.33\% $(n=10)$ of the patients presented treatment success, remaining asymptomatic. One patient 


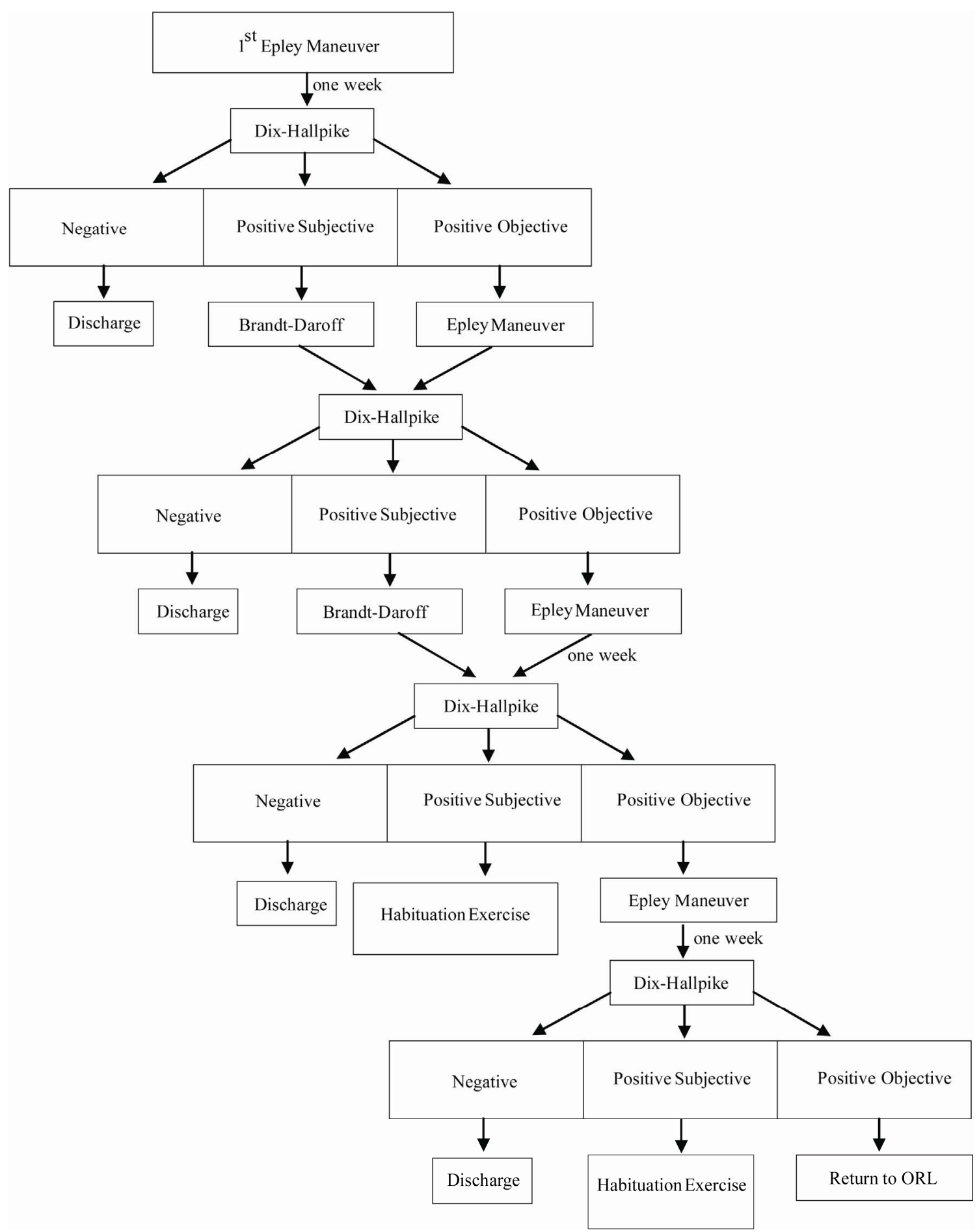

Enclosure I. Flow diagram for the treatment of BPPV.

(8.33\%) reported partial success and important improvement, and the last patient $(8.33 \%)$ was unable to return to finalize treatment.

During the evaluation performed two years after discharge, of the 10 patients who reported treatment success $(83.33 \%)$ and were asymptomatic, 75\% $(n=9)$ maintained treatment success, a fact demonstrating the efficacy of the repositioning maneuver up to two years after treatment in these cases of BPPV. Only two patients (16.66\%) reported current recurrence of vertigo, characterizing partial treatment success.

The data obtained were analyzed statistically by the 

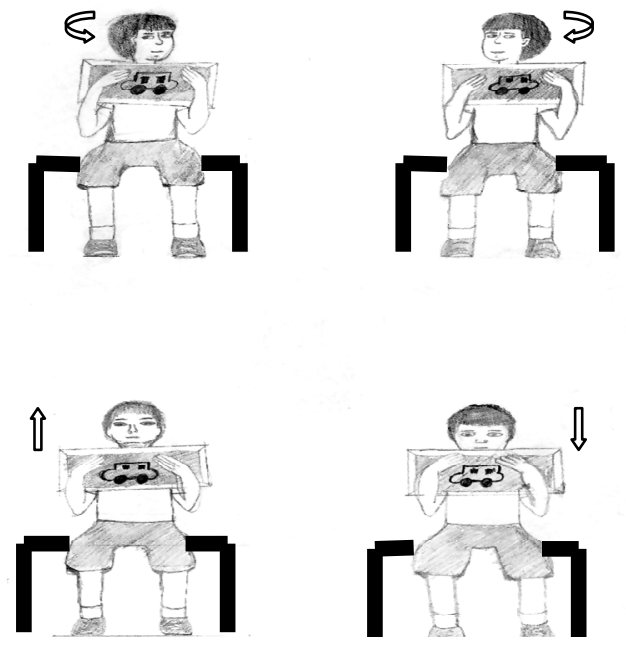

Figure 1. Demonstration of the exercises for the stimulation of the vestíbulo-ocular reflex. ${ }^{15}$

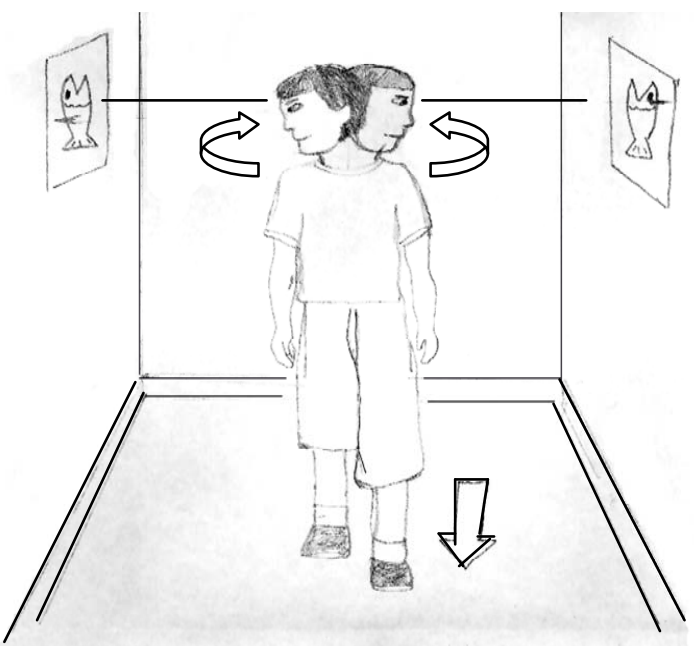

Figure 2. Demonstration of the exercises for the stimulation of the vestibulo-ocular and vestibulospinal reflexes.

chi-square test for categorical data, which revealed significant differences $(p<0.001)$ (Figure 3).

Paired pre- and post-treatment statistical analysis (Figure 4) revealed a statistically significant difference $(p<0.01)$, demonstrating treatment success after the repositioning maneuver. When the results obtained after the first treatment were compared to those obtained 24 months after treatment (Figure 5), no significant difference was observed $(p=0.99)$. Figure 6 shows a significant difference of results between the first treatment and the period of 2 years after it $(p<0.05)$, suggesting the therapeutic success of the repositioning maneuver.

The two patients who suffered relapses were women respectively aged 53 and 63 years who reported dizziness of the rotary type (vertigo) in sporadic crises of mild to
Initial visit - Please indicate below the sentence that best describes what you feel (Shepard et al. ${ }^{18}$ ):

1 ( ) (mild) Nonsignificant symptoms.

2 ( ) (moderate) Unpleasant symptoms.

3 ( ) (moderately severe) Being able to carry out the habitual tasks, but the symptoms interfere with external activities.

4 ( ) (severe) The symptoms interrupt the execution of habitual and external activities.

5 ( ) (serious) Currently on medical leave or had to quit his job due to the symptoms.

6 ( ) (incapacitating) Unable to work for more than one year or permanent disability.

Final visit - Please indicate below the sentence that best describes what you feel (adapted from Shepard et al. ${ }^{18}$ ):

1 ( ) (Therapeutic success) Asymptomatic.

2 ( ) (Partial success) Important improvement during the rest of the day after treatment.

3 ( ) (Relative success) Discrete improvement, symptoms defined and present.

4 ( ) (Unchanged) No improvement or worsening of symptoms.

5 ( ) (Failure) Worsening of symptoms after treatment.

Enclosure II. Assessment of discomfort.

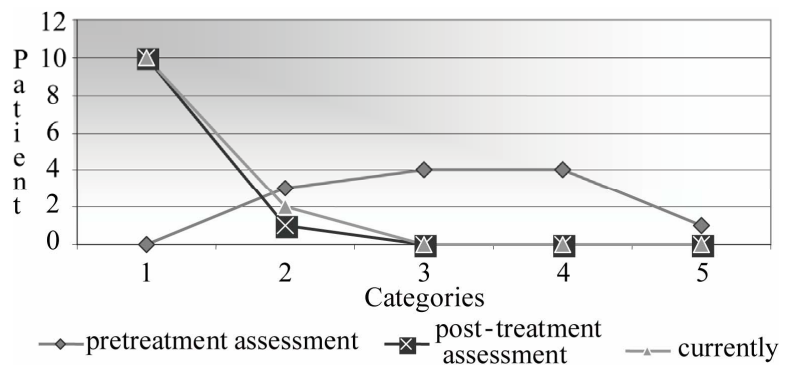

Figure 3. Assessment of patient discomfort in the pretreatment situation (1, mild; 2 , moderate; 3 , moderately severe; 4 , severe; 5 , serious; 6 , incapacitating) and after treatment, 2 years after the initial discharge or currently (1, treatment success; 2 , partial success; 3 , relative success; 4 , unchanged; 5 , failure).

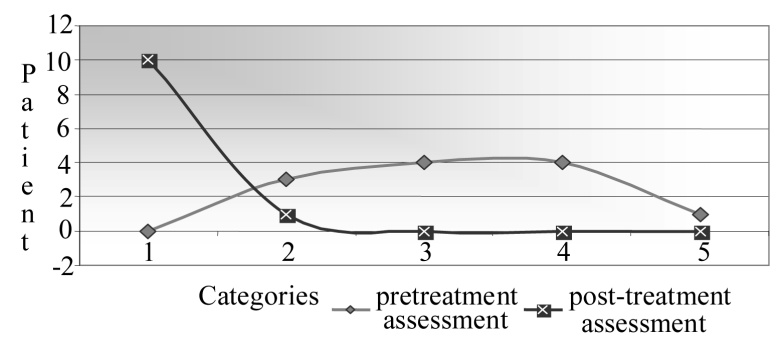

Figure 4. Assessment of patient discomfort in the pretreatment situation (1, mild; 2 , moderate; 3 , moderately severe; 4 , severe; 5 , serious; 6 , incapacitating) and after treatment, (1, treatment success; 2 , partial success; 3 , relative success; 4, unchanged; 5 , failure). 


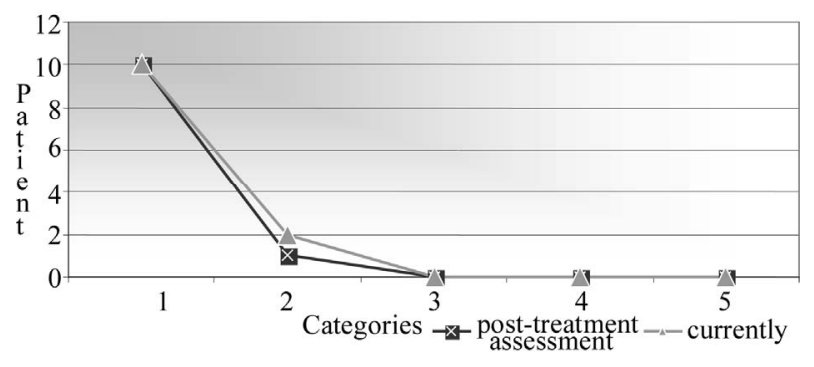

Figure 5. Assessment of patient discomfort in the posttreatment situation (1, treatment success; 2 , partial success; 3 , relative success; 4 , unchanged; 5 , failure) and 2 years after the initial discharge or currently (1, treatment success; 2 , partial success; 3 , relative success; 4 , unchanged; 5 , failure).

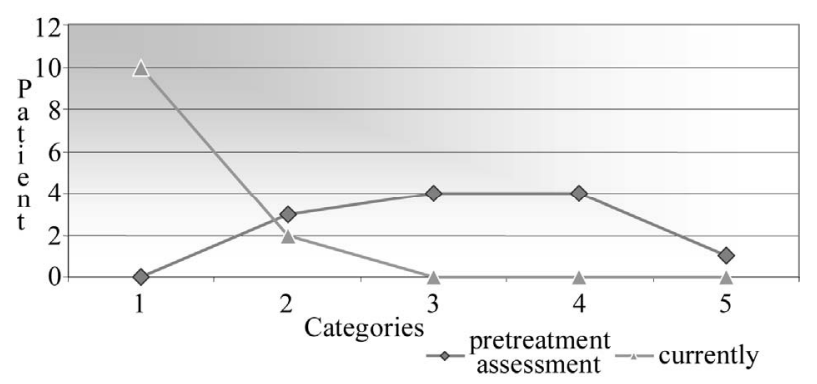

Figure 6. Assessment of patient discomfort in the pretreatment situation (1, mild; 2 , moderate; 3 , moderately severe; 4 , severe; 5 , serious; 6 , incapacitating) and 2 years after the initial discharge or currently (1, treatment success; 2 , partial success; 3 , relative success; 4 , unchanged; 5 , failure).

moderate intensity, with worsening of symptoms in the presence of abrupt head movements, a fact possibly characterizing a recurrence of BPPV.

Both patients also reported postural instability. In patient 1 , the recurrence of symptoms coincided with crises of arterial hypertension. The patient had excess body weight and presented cervicospinal alteration, which contraindicated the execution of physical activity. She was taking medications for obesity, depression and arterial hypertension. Because of the impossibility to perform physical exercises, the maneuvers for the treatment of BPPV were contraindicated. The cited exercises of vestibular compensation were indicated for VR and the patient presented improvement of symptoms two months after treatment. Patient 2 continued to follow the guidelines suggested after discharge, daily walks in particular. After recovering from an episode of the flu, she gradually returned to her physical activities and to the BrandtDaroff exercises. She was instructed to perform these exercises twice a day for three months, showing no symptoms after this period.

\section{Discussion}

In a study of a population of 87 patients, Cohen and
Jarabeck detected a significant rate of recurrence of frequency and intensity of vertigo after six months. Few of these patients reported residual vertigo, which did not require a new evaluation or treatment since these residual symptoms did not interfere with their daily routine [10].

Hain et al. detected a $17 \%$ rate of recurrence during the first four to six months after treatment, increasing to $47 \%$ of patients treated by the repositioning maneuver after five years of follow-up [11]. These results suggest that there is a cumulative increase of BPPV recurrence over time [11]. In the present study, although two patients suffered relapses, we may state that, for this specific study, the repositioning maneuver presented therapeutic success even two years after discharge, suggesting in a statistically significant manner that the sample of two years ago and the present sample remained unchanged, with the absence of BPPV symptoms.

Sidhar and Panda detected recurrence in 5\% of a group of 40 patients after six months of treatment, a rate that increased to $10 \%$ one year after discharge from treatment [17]. Maia et al. detected a $12.5 \%$ rate of recurrence one year after discharge in a group of eight patients [14]. The cases in question were treated again by repositioning maneuvers with therapeutic success, in agreement with the proposals of Epley, who stated that, even among patients correctly treated for BPPV, there may be a rate of recurrence of symptoms of 30 to $45 \%$ [9].

In the present study, $83.34 \%$ of the patients presented no recurrence of symptoms related to vertigo after discharge from treatment, whereas $16.66 \%$ of the patients did present recurrence, a fact that was not statistically significant for the sample studied. We observed rates similar to those reported by Maia et al. and Sidhar and Panda, who detected rates of 10 and $12.5 \%$, respectively, after one year of follow-up [14,17]. We did not find studies with a follow-up of two years; however, it should be pointed out that relapsing patients experienced symptoms starting one year and four months after discharge.

Comparison of patient symptoms before and after treatment and comparison of symptoms before treatment and current symptoms revealed statistically significant differences for both situations ( $p<0.01$ and $p<0.05$, respectively). There results were quantitated and analyzed statistically by qualitative assessment of symptoms before and after treatment, emphasizing the importance of this type of evaluation for the monitoring of treatment and the definition of discharge, since objective otoneurologic evaluation contributes little to the quantitation of symptoms before and after treatment of BPPV by providing normal results without specific findings for BPPV in caloric tests, thus being of little diagnostic help [14].

The patients with recurrent vertigo were older than 50 years, in agreement with other studies that attributed the 
greater involvement of older patients to a decline in the function of vestibular nuclei and of central vestibular pathways $[2,18,21]$. However, the age of the patients is not a factor that influences the rate of recurrence of symptoms [20]. The groups with recurrence presented the first symptoms starting one year and four months after discharge, as also observed by Hain et al. who reported that recurrence of symptoms in BPPV were mainly observed during the first two years after discharge [20]. The characteristics of the recurring symptoms in both patients clearly demonstrate, based on clinical history, recurrence of BPPV, which is the most common type of dysfunction of the vestibular system, characterized by sporadic crises of vertigo of short duration and variable intensity occurring when the head is moved in certain positions [1,21].

The first patient suffered recurrence of symptoms associated with crises of arterial hypertension, with hypertension being diagnosed after labyrinth crises treated in the hospital setting. The various vestibulopathies may be secondary to vascular disorders such as arterial hypotension or hypertension and arteriosclerosis [22]. Other factors contributing to vestibular disorders were the apparent excess weight and cervicospinal alteration that led to the contraindication of physical activities, with the patient requiring medications for obesity, depression and arterial hypertension. Cervicospinal alterations are an important contraindication of the repositioning maneuver, as well as a limitation of body movements, impairing vestibular compensation. Thus, the patients presented variables that impaired the maintenance of the bodily equilibrium obtained after the initial treatment, which probably induced recurrences.

The second patient had a milder complaint compared to the first and she continued to follow the guidelines received after discharge from the first treatment, especially daily walks. After suffering a cold, she presented recurrence of the characteristic symptoms of BPPV, although of mild intensity. The flu, colds, migraine and other factors affecting the health and general well-being of an individual impair the progression of treatment. Thus, the patient should first treat these disorders and then restart the program of vestibular treatment by means of repositioning maneuvers only after their cure. At the end of treatment with cold medications, the patient gradually resumed her physical activities and the BrandtDaroff exercises, as previously recommended. Although it can be effectively treated, BPPV is a disease that tends to recur and, most of the times the treatment indicated for the recurring symptoms is based on Brandt-Daroff exercises [11].

Questionnaires containing qualitative measures are of help for the assessment and monitoring of treatment for
BPPV and can be of help for the determination of the time of discharge after the initial treatment.

To this sample of patients results to a long-term follow-up were obtained for the treatment of the posterior canal BPPV based on repositioning maneuvers with highly effective. However, studies on larger populations of patients with BPPV are needed to characterize the real long-term efficacy of maneuvers in BPPV, contributing to a standardization of the rates of recurrence of symptoms.

\section{Conclusions}

Vestibular treatment of BPPV based on repositioning maneuvers is highly effective, with long-term results over a two year period of follow-up.

\section{REFERENCES}

[1] S. J. Herdman and R. J. Tusa, "Avaliação E Tratamento dos Pacientes Com BPPV,” In: S. J. Herdman, Ed., Reabilitação Vestibular, Manole, São Paulo, 2002, pp. 447471.

[2] R. S. M. Bittar, M. E .B. Pedalini, I. R. Y. Medeiros, M. A. Bottino and R. F. Bento, "Vestibular Rehabilitation in Children: Preliminary Study," Brasilian Journal of Otorhinolaryngology, Vol. 68, No. 4, 2002, pp. 496-499.

[3] C. B. Pereira and M. Scaff, "Benign Paroxysmal Positioning Vertigo,” Archives of Neuropsychiatry, Vol. 59, No. 2-B, 2001, pp. 466-470.

[4] J. C. Bulamarqui, C. A. H. Campos and O. M. Neto. "Epley’s Manouver for Benign Postural Paroxismal Vertigo: Systematic Review,” Acta Otolaryngology, Vol. 24, No. 1, 2006, pp. 15-22.

[5] S. Sridhar, N. Panda and M. Raghunathan, "Efficacy of Particle Repositioning Maneuver in BPPV: A Prospective Study” American Journal of Otolaryngology, Vol. 24, No. 6, 2003, pp. 355-360. doi:10.1016/S0196-0709(03)00069-3

[6] J. T. Vrabec, "Benign Paroxysmal Vertigo and Otolith Repositioning," Archives Otolaryngology and Head Neck Surgery, Vol. 124, No. 2, 1998, pp. 223-225.

[7] L. J. Teixeira and J. N. P. Machado, "Maneuvers for the Treatment of Benign Positional Paroxysmal Vertigo: A Systematic Review,” Brasilian Journal of Otorhinolaryngology, Vol. 72, No. 1, 2006, pp. 130-139.

[8] M. J. Ruckenstein, "Therapeutic Efficacy of the Epley Canalith Repositioning Maneuver," The Laryngoscope, Vol. 111, No. 6, 2001, pp. 940-945. doi:10.1097/00005537-200106000-00003

[9] J. M. Epley, “The Canalith Repositioning Procedure for Treatment of Benign Paroxysmal Positional Vertigo," Otolaryngology and Head Neck Surgery, Vol. 107, No. 3, 1992, pp. 399-404.

[10] H. S. Cohen and J. Jerabeck, "Efficacy of Treatments for Posterior Channel Benign Paroxysmal Positional Vertigo,” The Laryngoscope, Vol. 109, No. 4, 1999, pp. 
584-590. doi:10.1097/00005537-199904000-00012

[11] T. C. Hain, J. O. Helminski, I. L. Reis and M. K. Uddin, "Vibration Does Not Improve Results of the Canalith Repositioning Procedure," Archieves Otolaryngology and Head Neck Surgery, Vol. 126, No. 5, 2000, pp. 617-622.

[12] C. A. Moreno and K. J. Renaud, "Lãs Maniobras de Epley y Semont em el Tratamiento del Vértigo Postural Paroxí- stico Benigno,” Gaceta Medica de Mexico, Vol. 136, No. 5, October 2000, pp. 433-443.

[13] M. M. Ganança, H. H. Caovilla, F. F. Ganança, C. F. Ganança, M. S. L. Munhoz and M. L. G. Silva, "Benign Paroxysmal Positional Vertigo after Ménière Desease,” In: M. M. S. L. Munhoz, Ed., Typical and atypical Otoneurologic Clinical Cases, Atheneu, São Paulo, 2001, pp. 199-201.

[14] R. A. Maia, F. L. Diniz and A. Carlesse, "Treatment of Benign Paroxysmal Positional Vertigo with Repositioning Manevers,” Brasilian Journal of Otorhinolaryngology, Vol. 65, No. 5, 2001, pp. 612-616.

[15] E. B. Mantello, A. P. R. André, J. F. Colafêmina and M. L. Issac, "Bilateral Labyrinthine Hyporeflexy: Vestibular Rehabilitation Treatment," Proceedings of the 6th International Conference on Pediatric Otorhinolaryngology, Athens, Vol. 68, No. 5, 2004, pp. 633-731.

[16] I. D. S. Rivera, B. S. Zeigeboim and A. L. Jurkiewicz, "Vestibular Rehabilitation in Pregnant Women with Benign Paroxysmal Positional Vertigo: A Study of two
Cases,” Acta AWHO, Vol. 21, No. 2, 2003, 4-8.

[17] S. Sridhar, N. Panda and M. Raghunathan, "Efficacy of Particle Repositioning Maneuver in BPPV: A Pprospective Study," American Journal of Otolaryngology, Vol. 24, No. 6, December 2003, pp. 355-360. doi:10.1016/S0196-0709(03)00069-3

[18] N. T. Shepard, S. A. Telian and M. Smith-Weelock, "Habituation and Balance Retraining Therapy: A Retrospective Review,” Neurologic Clinics, Vol. 8, No. 2, 1990, pp. 459-475.

[19] M. M. Ganança, "Rational Pharmacotherapy of the Vertigo,” In: M. M. Ganança, Ed., Is Vertigo Have Cure? Lemos Editorial, São Paulo, 1998, pp. 227-235.

[20] C. F. Ganança, C. F. Dias and M. M. Ganança, "Nutritional Guidance and Changes of Habit of Vertiginous Patient,” In: M. M. Ganança, Ed., Therapeutic Strategies in Otoneurology, Atheneu, São Paulo, 2001, pp. 55-66.

[21] M. Fetter, “Dysfunctions of the Vestibular System,” In: S. J. Herdman, Ed., Vestibular Rehabilitation, Manole, São Paulo, 2002, pp. 91-101.

[22] M. M. S. L. Munhoz, M. M. Ganança, H. H. Caovilla, L. G. Silva, F. F. Ganança and C. F. Ganança, "Benign Paroxysmal Positional Vertigo Ductolitiasis of the Lateral Semicircular Canal on the One Hand and Cupulolitiasis of the Lateral Semicircular Canal Side of,” In: M. M. S. L. Munhoz, Ed., Typical and Atypical Otoneurologic Clinical Cases, Atheneu, São Paulo, 2001, pp. 195-196. 